Article

\title{
Ultra-Stable UiO-66 Involved Molecularly Imprinted Polymers for Specific and Sensitive Determination of Tyramine Based on Quartz Crystal Microbalance Technology
}

\author{
Chi-Xuan Yao ${ }^{1}$, Ning Zhao ${ }^{2}$, Jing-Min Liu ${ }^{2}$, Guo-Zhen Fang ${ }^{1}$ and Shuo Wang ${ }^{1,2, *}$ \\ 1 State Key Laboratory of Food Nutrition and Safety, Tianjin University of Science \& Technology, \\ Tianjin 300457, China; yaochixuan@mail.tust.edu.cn (C.-X.Y.); fangguozhen@tust.edu.cn (G.-Z.F.) \\ 2 Tianjin Key Laboratory of Food Science and Health, School of Medicine, Nankai University, Tianjin 300071, \\ China; 15891783438@163.com (N.Z.); liujingmin@nankai.edu.cn (J.-M.L.) \\ * Correspondence: wangshuo@nankai.edu.cn; Tel.: +86-22-85358445
}

Received: 14 January 2020; Accepted: 27 January 2020; Published: 31 January 2020

\begin{abstract}
A rapid method was developed to determine the content of tyramine in food on the basis of the combination of molecular imprinting technique and the metal-organic frameworks. We developed the new molecular imprinted polymers based on metal-organic frameworks UiO-66 (named UiO-66@MIPs) as the sensing recognition element, the non-molecular imprinted polymers based on UiO-66 (named UiO-66@NIPs) was synthesized according the same steps without tyramine for comparison. The characterization of obtained UiO-66@MIPs was investigated through a series of characterization experiments. The results indicated that the octahedral shaped UiO-66 was encapsulated in the sol-gel polymer film, with a desirable thermal stability and possessed a specific surface area (SSA) of $994.3 \mathrm{~m}^{2} \cdot \mathrm{g}^{-1}$. The imprinting factor of the UiO-66@MIPs for tyramine was 1.956 in static experiment. This indicates the synthesized UiO-66@MIPs have outstanding performance compered to UiO-66@NIPs on the static adsorption quantity and selective adsorption affinity. It's to make use of advantages of the synthetic materials to develop a quartz crystal microbalance (QCM) sensor for the sensitive detection of tyramine. The detection limit of the system was $61.65 \mu \mathrm{g} \cdot \mathrm{L}^{-1}$ within measurable concentration range from 80 to $500 \mu \mathrm{g} \cdot \mathrm{L}^{-1}$. The prepared QCM sensor was verified in selectivity and application. The UiO-66@MIPs possess good behavior on selectivity, absorptivity, and chemical stability, so the UiO-66@MIPs achieve accurate and rapid trace detection of biogenic amines in food combining with the quartz crystal microbalance.
\end{abstract}

Keywords: metal-organic frameworks; UiO-66; molecularly imprinted polymers; quartz crystal microbalance; tyramine

\section{Introduction}

Biogenic amines are a class of small molecular nitrogen compounds with biological activity. The existence of a low content of biogenic amine plays an important physiological role in animals and plants [1]. Tyramine is metabolized by monoamine oxidase (MAO) [2] and is common in biogenic amines, which widely exists in fermented food or other animal-origin food matrices [3] and it has a great impact on human health [4]. Food allergens can cause a series of adverse reactions and impair human health. After excessive intake, excess tyramine contributes to an enhanced pressor effect of tyramine in human body [2]. Even, elevated blood pressure and other potential dangerous could take place following exposure to normal amounts of tyramine when using MAO inhibitors (MAOI), due to its inhibition of reducing tyramine [5]. We can see that the incidence of hypertension is closely 
related to the level of tyramine in the human body [6]. To take the scientific approach a step further for tyramine, it is a vital research work to measure the level of tyramine quickly and sensitively.

Some testing means, such as high-performance liquid chromatography [7] and capillary electrophoresis detection [8], required extra complicated pre-treatment processes. These testing means are not conducive to carry out spot quick detection, which are limited to the requirement of skilled technicians and sophisticated equipment. Enzyme-linked immunosorbent assay [9] (ELISA) should be determined under strict experimental condition, it will lead to inaccurate results in extreme conditions. Therefore, it is integral for the establishment of a rapid and accurate method to measure trace concentration of tyramine in food samples, reducing the risk of the control system on food safety [10].

The molecular imprinted polymers (MIPs) have the molecular recognition sites of the template molecule and, thus, obtained specific cavities for this template [11]. MIPs are workable to maximize the selectivity and sensitivity of detection sensors as recognition elements [4]. MIPs are widely used in the environmental [12], agricultural [13], food science [14], and pharmaceutical industries [15]. With the development of science and technology and the appearance of advanced instruments, the relatively homogeneous MIPs could not satisfy the growing technological requirements [16]. Recently, the researchers have turned their eyes to the molecular imprinted polymers and their hybrids based on nanomaterials due to their excellent performance in the development of sensors, hybridization MIPs, and the nanomaterial would endow some new functions to improve the performance of nanosensors [4,17-22]. Özkan et al. [4] reported a surface plasmon resonance (SPR) nanosensor incorporated MIPs and the nanocomposites of Ag@AuNPs nanoparticles/hexagonal boron nitride (HBN) nanosheets to measure the level of etoposide in urine samples, the linear range of this SPR sensor was $0.001-1.00 \mu \mathrm{g} \cdot \mathrm{L}^{-1}$ and the detection limit (LOD) was $0.00025 \mu \mathrm{g} \cdot \mathrm{L}^{-1}$. Kran et al. [17] reported an electrochemical sensor to assess methyl parathion via the hybrids of MIPs and nanomaterials, this sensor was relative stable in 60 days. Atar et al. [19] reported an electrochemical sensor to determine cypermethrin based on the hybrid material of MIPs/core-shell nanoparticles/HBN nanosheets, the linearity range was from $1.0 \times 10^{-13}$ to $1.0 \times 10^{-8} \mathrm{~mol} \cdot \mathrm{L}^{-1}$ and the LOD of this sensor was $3.0 \times 10^{-14} \mathrm{~mol} \cdot \mathrm{L}^{-1}$. Diethylstilbestrol (DES) is one of synthetic environmental hormones, it is quite stable and high toxicity that may be found in the industrial waste [20]. Yola et al. [21] established a molecularly imprinted sensor based on gold nanoparticles combined with two-dimensional-HBN nanosheets for DES detection. They also prepared a sensor of bilirubin measurement based on the nanocomposites of molecular imprinted polymers and functionalized carbon nitride nanotubes, this sensor was low cost and it could be used in routine analysis without disturbance [22]. Also, the molecularly imprinted technology could be coupled with quantum dots to detect a biochemical marker called cTnI of acute myocardial infarction (AMI) [23] and epinephrine [24]. Phenylethanolamine A (PEA) may cause acute toxic effects so that the researchers developed a kind of nanocomposite supported MIP materials based on Ru@Au NPs/carbon nitride nanotubes/graphene quantum dots to detect the level of PEA, the nanosensor was high selectivity and recovery even if in presence of other competitive agents in real samples.

The quartz crystal microbalance (QCM) sensor is famous for its outstanding performance and applied in some respects of our social life [25-28], which has been used as a transducer of sensors. There was negative correlation between the binding mass of target increases and the oscillation frequency [29]. In recent years, the QCM sensor combined with molecularly imprinting polymers (MIPs) has been applied to multipurpose detection [29-31]. MIPs improve the selectivity of the normal QCM sensor for target molecule. Meanwhile, MIPs can concentrate the target molecules to enhance the sensitivity of the QCM sensors due to their specific cavities for target. For these reasons, the MIPs-QCM sensors have been greatly developed in the last few years.

Metal-organic frameworks (MOFs) are a class of three-dimensional porous crystalline compounds formed by self-assembly of metal ions or clusters with organic ligands. Metal-organic frameworks (MOFs) stand out for large specific surface area, tunable pore diameter, well-defined structure and 
high micropore volume, entrusting MOFs with huge potential in correlative technological applications, gas storage [32] and separation [33], sensing [34], catalysis [35], and drug delivery [36]. Among these porous MOFs, $\mathrm{UiO}-66$ is a kind of $\mathrm{MOF}$ that is composed of inorganic nodes $\left(\mathrm{Zr}_{6}\right.$-oxyhydroxide clusters) and organic linkers (1,4-benzenedicarboxylate, BDC). It was out of the ordinary due to its high constancy in common solvents [37] and high hydrothermal stability up to $500{ }^{\circ} \mathrm{C}$ [38]. The morphology and uniform sizes of UiO-66 can be adjusted by adding acid [39] and controlling the concentrations of reagents and modulators [40].

UiO-66 owned excellent chemical and thermal stability in water and can be well applied to the adsorption of various organic molecules [41]. The regulatable pore size of UiO-66 is one of its superior advantages as an adsorbent [42]. However, the adsorption range is broad-spectrum, target molecules would be not absorbed efficiently and selectively. Realizing the material functionalization of UiO-66 surface is an effective method to enhance adsorption capacity [43]. Therefore, it is necessary to improve the adsorption efficiency and specificity of tyramine on UiO-66 materials by molecular imprinting technology. The relatively high adsorption capacity of MOFs and the rapid recognition ability of imprinted materials to target molecules can be obtained by introducing high specific surface area metal-organic frameworks (MOFs) as the matrix of molecular imprinting.

In this work, acetic acid promoted UiO-66 was the core of the molecular imprinted polymers to for tyramine detection via a sol-gel process. Then the selective core-shell architecture polymers (UiO-66@MIPs) are used as recognition elements in the fabrication of QCM biosensor toward the target compound. The preparation process of the sensor is shown in Scheme 1. Specifically, this method combined the merits of surface molecularly imprinting technology and metal-organic frameworks. The surface-imprinted polymers also provide ideas and directions for the efficient adsorption of target compounds by quartz crystal microbalance in practical complex samples. This paves the way for the development of a new kind sensor for tyramine. The UiO-66@MIPs material was integrated within quartz crystal microbalance device to determine the trace content of tyramine for the first time.

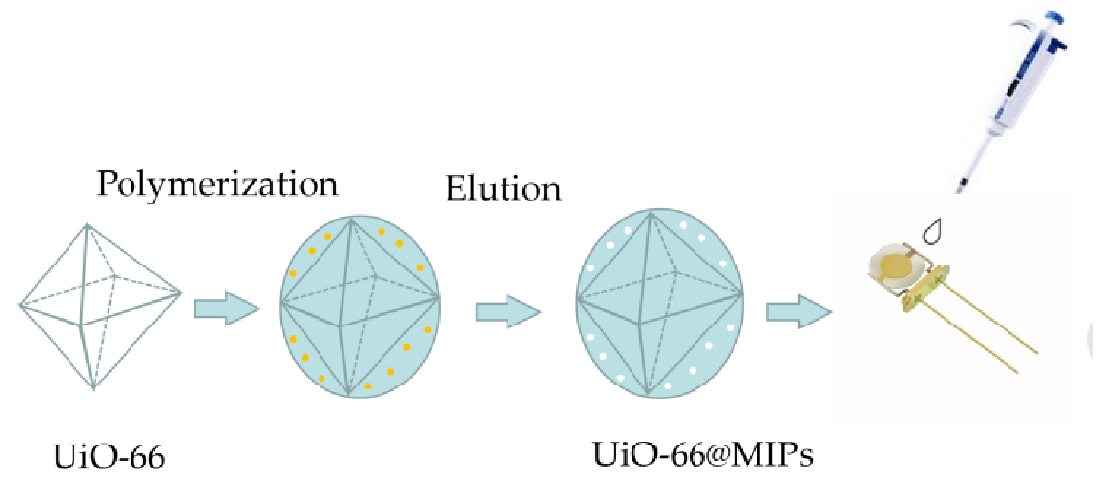

Scheme 1. The preparation process of the nanocomposite and quartz crystal microbalance (QCM) Pt electrode.

\section{Material and Methods}

\subsection{Instruments and Reagents}

Chemicals for the experiments were from Shang Hai Aladdin Biochemical Technology Co., Ltd., Shang Hai, China: zirconium tetrachloride (98\%), acetic acid (ACS, $\geq 99.7 \%)$, and terephthalic acid (99\%) were used for UiO-66 synthesis. Tyramine (98\%), 3-Aminopropyl triethoxysilane (APTES, 99\%), tetraethyl orthosilicate (TEOS, $99.99 \%$ metals basis), and ammonium hydroxide $\left(25-28 \%\right.$ in $\mathrm{H}_{2} \mathrm{O}$ ) were also used in the MIP synthesis. Doubly distilled water (DDW, $18.2 \mathrm{M} \cdot \mathrm{cm}^{-1}$ ) was obtained from a Water Pro water purification system (Labconco, Kansas, MO, USA). Electrochemical experiments were performed with an electrochemical workstation PAR 2273 (EG\&G) associated with a QCM (QCA922, Seiko EG\&G) at room temperature, with a $9 \mathrm{MHz}$ AT-cut quartz crystal plated with platinum electrode. 
UiO-66@MIP or NIP polymers were holding by Teflon that ensures that one side of the Pt electrode can only connect to the measured liquid while the other side is insulated.

\subsection{Synthesis of UiO-66 and UiO-66@MIPs/NIPs}

UiO-66 was obtained by solvothermal synthesis method [44]. The nanoparticles of molecular imprinted polymers based on UiO-66 (UiO-66@MIPs) were prepared with UiO-66 through sol-gel molecularly imprinted polymerization. The artificial materials were made of the functional monomer of APTES and the cross-linker of TEOS toward tyramine ("template") as schematically. Tyramine (100.0 mg) was firstly put into $5.0 \mathrm{~mL}$ ethanol with fully dissolution. APTES $(0.687 \mathrm{~mL})$ was added into the reaction system under continuous mixing. The prepolymer formed after stirring $30 \mathrm{~min}, 200.0$ $\mathrm{mg}$ of UiO-66 and $0.664 \mathrm{~mL}$ of TEOS were added into above mixture to continue the mixing process. Finally, $0.4 \mathrm{~mL}$ of $3.0 \mathrm{~mol} \cdot \mathrm{L}^{-1}$ aqua ammonia participated in reaction to catalyze the formation of highly crosslinked products. It took $24 \mathrm{~h}$ for polymerization process and the polymers were aged for $10 \mathrm{~h}$ under vacuum drying. The obtained polymers were eluted ethanol and water (1:1) until tyramine would be not detected by ultraviolet spectrophotometer, then dried under vacuum overnight. The nanoparticles of non-molecular imprinted polymers based on UiO-66 (UiO-66@NIPs) were synthesized following the same step as UiO-66@MIPs, only without adding template molecules.

\subsection{Adsorption of Tyramine.}

The adsorption method was conducted as follows. For static experiment, UiO-66@MIPs or UiO-66@NIPs $(4.0 \mathrm{mg}$ ) was mixed with $2.0 \mathrm{~mL}$ buffer (phosphate buffered saline, $\mathrm{pH}=7.4$ ) containing different concentrations of tyramine $\left(5.0-140 \mathrm{mg} \cdot \mathrm{L}^{-1}\right)$ in microcentrifuge tubes. The mixture was shaken for $12 \mathrm{~h}$ and centrifuged twice at 12,000 rpm for $15 \mathrm{~min}$. For adsorption kinetic experiment, 4 mg MIPs or NIPs was accurately weighed and placed in $2.0 \mathrm{~mL} 100 \mathrm{mg} \cdot \mathrm{L}^{-1}$ tyramine PBS standard solution, shaking for $10 \mathrm{~min}$ to $12 \mathrm{~h}$ at room temperature. The remaining tyramine in each supernatant fluid was estimated by the ultraviolet spectrophotometer at $225 \mathrm{~nm}$ to examine. The adsorption property of UiO-66@MIPs $\left(Q, \mathrm{mg}^{-1} \mathrm{~g}^{-1}\right)$ was calculated by Equation 1:

$$
Q=\left(C_{i}-K \times C_{f}\right) \times V / W
$$

where $C_{i}$ is the original concentration of tyramine in PBS solution $\left(\mathrm{mg} \cdot \mathrm{L}^{-1}\right), C_{f}$ is the final concentration of tyramine after adsorption (mg. $\left.\mathrm{L}^{-1}\right), K$ is dilution multiple in detection, $W$ is the amount of polymers (mg), and $V$ represented the volume of adsorbed solution (L).

\subsection{QCM Measurements}

Before being modified, quartz crystal gold electrode should be cleaned in ethanol by ultrasound, and let it soak in a new "piranha" solution $\left(30 \% \mathrm{H}_{2} \mathrm{O}_{2} /\right.$ concentrated $\left.\mathrm{H}_{2} \mathrm{SO}_{4}=1: 3, V / V\right)$ for $5 \mathrm{~min}$. After washing thoroughly, the quartz crystal Pt electrode was dried with nitrogen and assembled into the PTFE fixator. $10 \mathrm{mg}$ of UiO-66@MIPs or UiO-66@NIPs was accurately weighed and mixed into 2.0 $\mathrm{mL}$ dichloromethane (containing $2.0 \mathrm{mg}$ polyvinyl chloride) in centrifugal tubes, respectively. The mixture was uniformly distributed by vortex oscillation and ultrasound until a uniform suspension was obtained. The mixture $(5.0 \mu \mathrm{L})$ would be uniformly coated on Pt electrode layer. The modified electrode layer was dried and kept at room temperature, fixed in quartz crystal microbalance prior to use.

The assembled sensor was immersed in PBS buffer $(\mathrm{pH}=7.4,25 \mathrm{~mL})$ under magnetically stirring at $200 \mathrm{rpm}$ until the initial value of resonant frequency $\left(f_{0}\right)$ was obtained after reaching equilibrium. Minim tyramine solution was transferred to the cell, a new stable frequency change $(f)$ was obtained after adsorption equilibrium of target by $\mathrm{QCM}$ sensor. After each detection process, the modified $\mathrm{Pt}$ electrode was eluted by $1.0 \mathrm{~mol} \cdot \mathrm{L}^{-1} \mathrm{HCl}$ and deionized water up to the frequency change become 
stable again. We use this reusable modified Pt electrode to carry out the successive determination and every single experiment was repeated at least three times.

\subsection{Sample Preparation}

Standard addition technique was utilized for the determination of tyramine in terms of validation. Soy sauce was chosen as actual sample to evaluate the practical application value of UiO-66@MIPs-QCM sensor for tyramine measurement. Soy sauce was purchased from the native market and added tyramine standard solution of $200 \mu \mathrm{g} \cdot \mathrm{L}^{-1}, 300 \mu \mathrm{g} \cdot \mathrm{L}^{-1}$ and $400 \mu \mathrm{g} \cdot \mathrm{L}^{-1}$, separately. The added samples were extracted and degreased to remove unnecessary fat and protein. Through $0.22 \mu \mathrm{m}$ filtration, the prepared samples were detected by the prepared QCM sensors. $\Delta f$ was taken notes for analysis until the change of frequency was less than $1\left(\Delta f=f_{0}-f\right)$.

\section{Results and Discussion}

\subsection{Characterization}

MOFs were provided with permanent porosity after removal of guest molecules by activation. Moreover, MOFs have the high porosity of the material skeleton, low density and large specific surface area [45]. UiO-66 has productive chemical stability [46], therefore its crystalline structure can be maintained under strong acid and alkaline corrosion environment. Based on the above-mentioned advantages, we choose UiO-66 as the supporter of molecularly imprinted polymers.

Acetic acid promoted UiO-66 presented more regular octahedron crystalline shape than the synthetic samples without acid regulator [39], and the particle was uniform size with good dispersion performance and no agglomeration, which the average particles size is about $734 \mathrm{~nm}$ (Figure 1A). After polymerization, it is obvious that the clear octahedral structure of UiO-66 had disappeared in Figure 1B. There is a film wrapped around the surface of UiO-66 crystalloid, the aggregation actually occurs between UiO-66 crystals. The imprinted layer was wrapped on the outer of UiO-66 crystals, the size of particles becomes larger-about $757 \mathrm{~nm}$. The obtained larger nanoparticles indicated that the MIPs layer was successfully formed outside UiO-66.

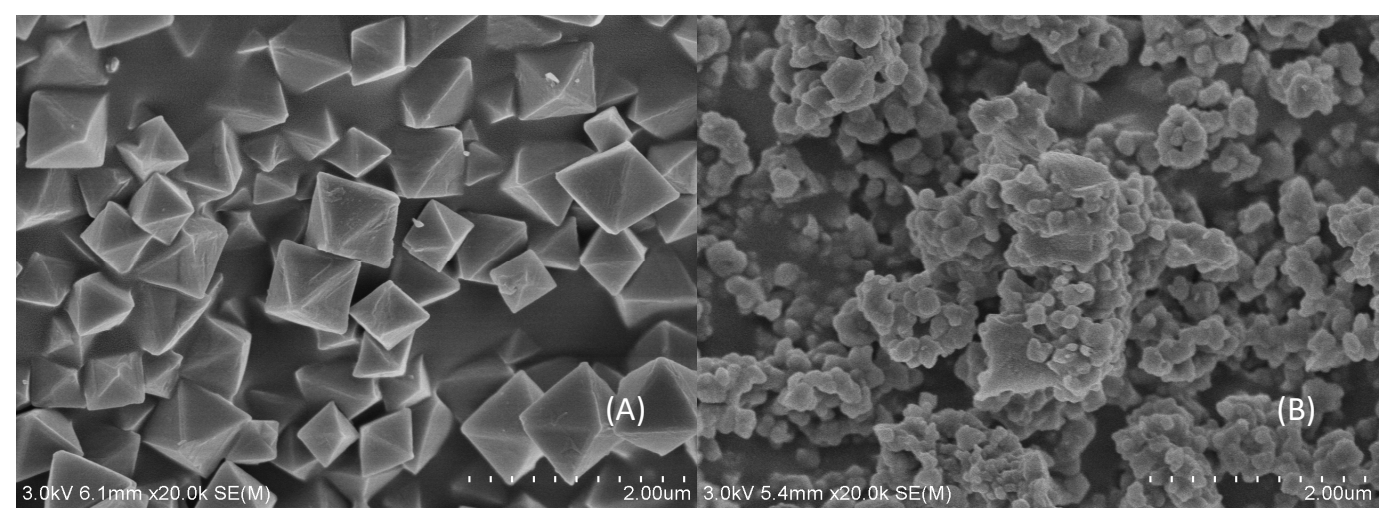

Figure 1. SEM images of synthesis samples: (A) UiO-66 and (B) UiO-66@MIPs (molecular imprinted polymers).

Figure 2A is the XRD spectra of MOFs and UiO-66@MIPs. The XRD patterns of UiO-66 show the strong and narrow typical UiO- 66 characteristic peaks, which at $2 \theta=7.35^{\circ}$ and $8.50^{\circ}$, indicating well-quality crystal structures. The patterns of UiO- 66 were in good agreement with the previous literature report [47]. For UiO-66@MIPs, we can observe clearly after surface-imprinting process, the XRD peaks intensity of UiO-66 almost decreased, forming the coating polymerization successfully.

The size and crystallinity of UiO-66 can be adjusted by changing the proportion of acetic acid during the synthesis process. The $\mathrm{N}_{2}$ adsorption graph (Figure $2 \mathrm{~B}$ ) shows the isothermal adsorption curve of UiO-66 we prepared. It can be seen that the synthesized UiO-66 is a typical type I adsorption isotherm, 
which indicates the acetic acid promoted UiO-66 belongs to microporous material. At the same time, the specific surface area of UiO-66@MIPs was $19.258 \mathrm{~m}^{2} \cdot \mathrm{g}^{-1}$, much lower than the unmodified UiO-66 $\left(994.3 \mathrm{~m}^{2} \cdot \mathrm{g}^{-1}\right)$. These characterizations contributed to the successfully prepared UiO-66. The results demonstrate the existence of a surface-imprinted layer.
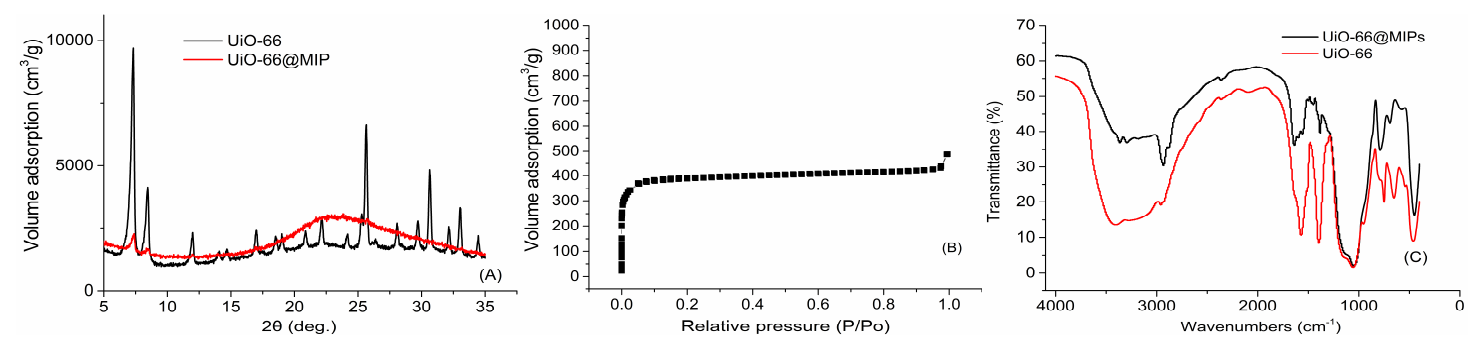

Figure 2. The Characterization of the materials: (A) the XRD patterns of UiO-66 and UiO-66@MIPs; (B) the $\mathrm{N}_{2}$ patterns of UiO-66; and (C) the FTIR patterns of UiO-66 and UiO-66@MIPs.

Fourier transform infrared spectroscopy (FTIR) is a powerful method to characterize the properties of organic groups. UiO-66 and UiO-66@MIPs were characterized by FTIR (Figure 2C). The pattern of UiO-66 shows the carbon-carbon double bond and carboxyl group structure of the phenyl ring skeleton from terephthalic acid. Compared with UiO-66, MIPs have weak $C=C$ vibrations at $1620 \mathrm{~cm}^{-1}$, intensity of the strong absorption peaks weakened at $3400 \mathrm{~cm}^{-1}$ and $1570 \mathrm{~cm}^{-1}$, suggesting that polymerization and hydrolysis reaction happened on the surface of UiO-66 and the existence of molecularly imprinted polymers.

\subsection{Adsorption of Tyramine-MIPs}

As shown in Figure 3A, the adsorption capacities of the prepared materials (UiO-66@MIPs and UiO-66@NIPs) increased with tyramine concentration. In the initial stage, the equilibrium adsorption capacities increased rapidly, the adsorption capacity of MIPs to tyramine was always higher than that of NIPs. Then the adsorption capacities increased slowly until they reached saturation. UiO-66@MIPs have better adsorption capacity for tyramine than UiO-66@NIPs. The equilibrium adsorption capacity

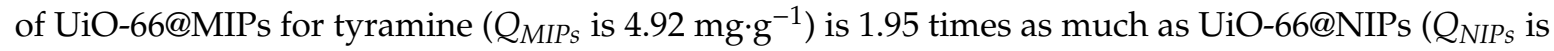
$\left.2.51 \mathrm{mg} \cdot \mathrm{g}^{-1}\right)$ at low level $\left(80 \mathrm{mg} \cdot \mathrm{L}^{-1}\right)$. UiO-66@MIPs have better adsorption capacity for tyramine than non-imprinted polymers. This may be due to better matching of recognition sites and templates on imprinted surfaces, allowing the imprinted layer to absorb more template molecules. On the other hand, based on the surface imprinting technology, the thinner the thickness of imprinted shell is, the faster the mass transfer speed UiO-66@MIPs it has, while UiO-66@NIPs are mainly non-specific adsorption.
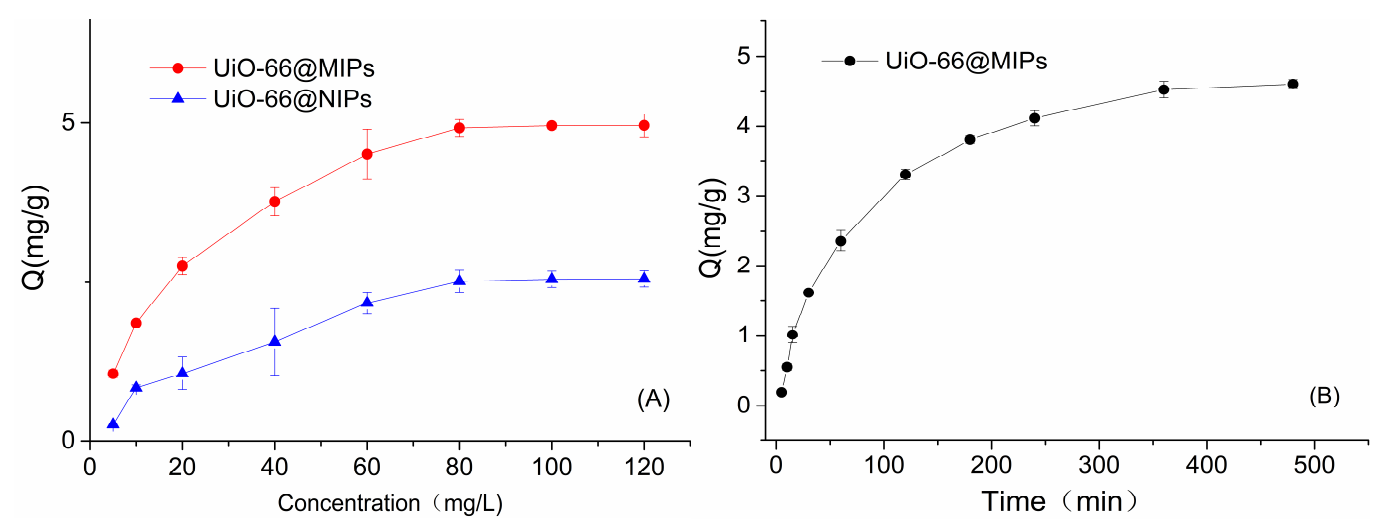

Figure 3. Binding isotherm of UiO-66@MIPs and UiO-66@NIPs for tyramine: (A) static adsorption curve and (B) dynamic adsorption curve. 
Furthermore, the dynamic adsorption kinetic of UIO-66@MIPs was also studied (Figure 3B). The adsorption time of UIO-66@MIPs was investigated under the same concentration. The result shows that the adsorption capacity reached $1.78 \mathrm{mg} \cdot \mathrm{g}^{-1}$ at $30 \mathrm{~min}$, basically reaching equilibrium state in $4 \mathrm{~h}$. The adsorption rate of the prepared molecularly imprinted polymers was relatively fast, mainly because the mass transfer rate of UiO-66@MIPs was high due to the comparative soft structure of the sol-gel molecularly imprinted polymer, the template molecules are more easily adsorbed.

\subsection{The Performance of QCM Sensor}

UiO-66@MIPs and polyvinyl chloride (PVC) are the main components of modified membranes on the surface of the gold electrode. Under a certain amount of coating $(10.0 \mu \mathrm{L})$, different amounts of MIPs added would affect the imprinted binding sites of the modified membranes. Therefore, the amount of imprinted polymer should be maximized while the sensitive film is closely modified on the quartz wafer. In $500 \mathrm{mg} \cdot \mathrm{L}^{-1}$ tyramine standard solution, the frequency changes of the sensors were measured under different coating proportions of PVC and polymers. From Figure 4A, the frequency was up to maximum variation when UiO-66@MIPs was four times the quantity of PVC. Meanwhile, the frequency of the sensor based on UiO-66@MIPs became stable gradually in PBS buffer, target solution was dropped into the buffer and the frequency of the sensor decreased. Also, we can find that the sensor handily took up tyramine in $10 \mathrm{~min}$ (Figure 4C), reached the constant value after $16 \mathrm{~min}$.
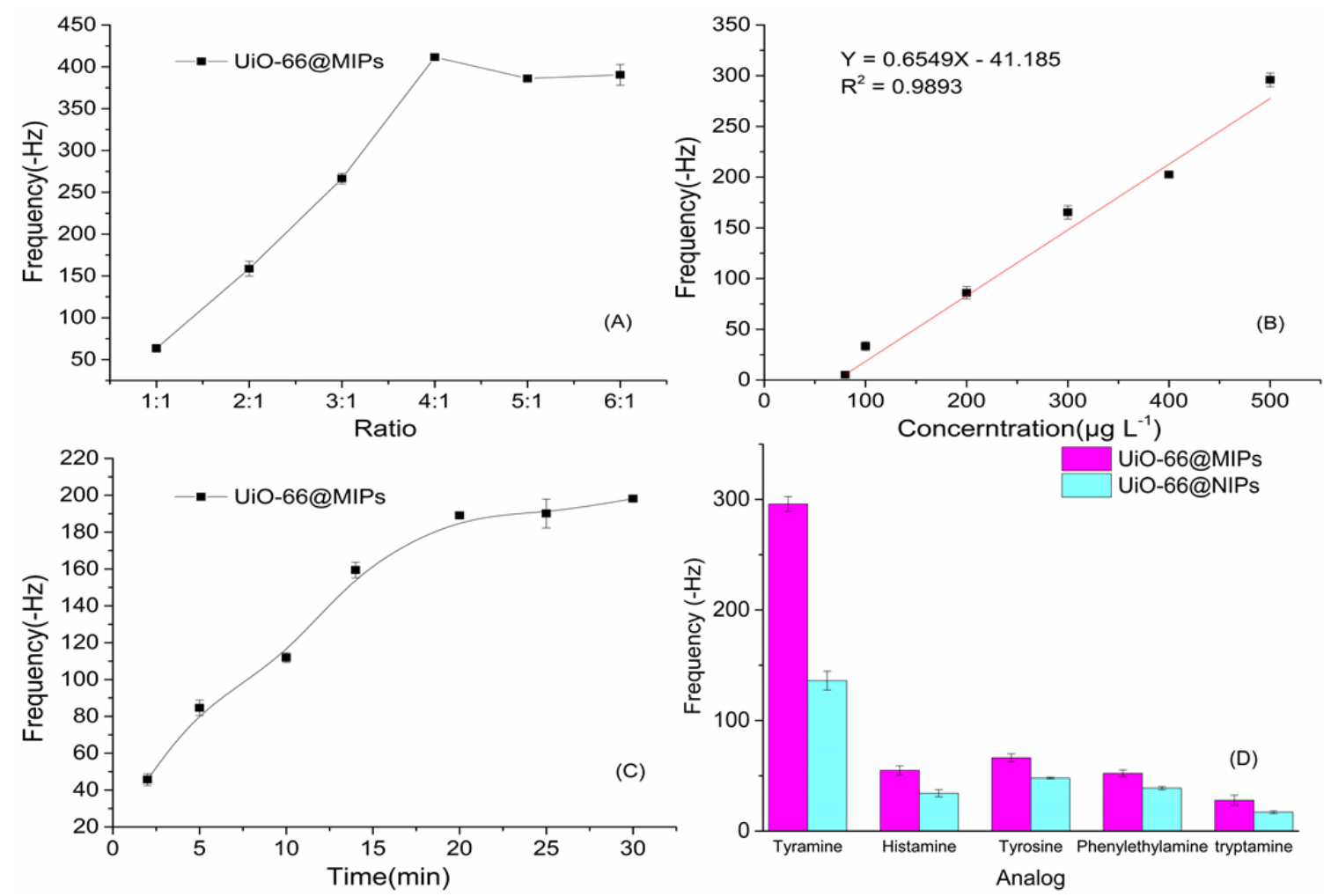

Figure 4. The performance of UiO-66@MIPs QCM sensor: (A) Effects of different ratios of PVC to MIPs on QCM sensors; (B) Frequency changes of UiO-66@MIPs QCM sensor in tyramine standard solutions; (C) Response time of the QCM sensor; and (D) The selective performance of UiO-66@MIPs/UiO-66@NIPs QCM sensors.

Quartz crystal microbalance sensors based on UiO-66@MIPs was used to evaluate the binding capacity of tyramine at different concentrations. As shown in the Figure $4 \mathrm{~B}, \Delta f$ value increased with increasing concentrations of tyramine. This could be attributed to the binding sites of UiO-66@MIPs. The generated sites could fix the target molecule specifically ascribed to porous structure and effective chemical groups on MIPs film. The frequency change of UiO-66@MIPs sensor response is linear with 
the concentration of tyramine in range of $80-500 \mu \mathrm{g} \cdot \mathrm{L}^{-1}$ under certain condition. In addition, the LOD was $61.65 \mu \mathrm{g} \cdot \mathrm{L}^{-1}$.

The specificity is a major challenge for rapid detection methods to ensure the accuracy. The potential competitive agents were unavoidable as the coexisting substances during the analysis. Tyramine, histamine, and tryptamine are the products after tyrosine decarboxylation [48]. Histamine, tryptamine, $\beta$-phenylethylamine, and tyramine all belong to monoamines and were common in food samples [49-52]. Thus, we chose histamine, tryptamine, $\beta$-phenylethylamine, and tyrosine to verify selectivity of the sensors. Under the same concentration, we can see the UiO-66@MIPs sensor has the highest response to tyramine from Figure $4 \mathrm{D}$, but also has weak response to other compounds. Some functional groups similar to that of tyramine can interact with the binding sites in the cavities of polymer particles on the modified membranes. However, due to the structural differences between them, these competitive molecules could not fully enter the imprinted cavities and combine the active binding site in the cavities, which results in the response of every analog is weaker than that response of tyramine. For UiO-66@NIPs sensor, either tyramine or other compounds, the responses were very small due to the no suitable sites for tyramine. Further, we defined the imprinting factor (IF) for selectivity estimate in QCM experiment,

$$
I F_{Q C M}=\Delta f_{M I P} / \Delta f_{N I P}
$$

where $\Delta f_{\text {MIP }}$ and $\Delta f_{\text {NIP }}$ are respective response frequency variations of the five analytes. The detection results that the impact factor of tyramine was 2.17, obviously higher than the result of any other analog (the impact factors of histamine, tyrosine, tryptamine, $\beta$-phenylethylamine, and tryptamine were 1.60, $1.38,1.34$, and 1.81 , separately), indicating that the UiO-66@MIPs-QCM sensor had excellent specificity for tyramine. This showed the UiO-66@MIPs-QCM sensor was of excellent selectivity by frequency change, and also proves the successful application of molecularly imprinted technology and QCM technology.

\subsection{Evaluation of sample analysis and comparison of the methods}

After sample pretreatment, the content of tyramine in soy sauce samples, which were added at three concentrations, was tested by UiO-66@MIPs-QCM sensor. The recovery for the detection results ranged from $86.74 \%$ to $93.62 \%$ in Table 1, expressing that as-prepared UiO-66@MIPs-QCM sensor could be used in practical application for accurate tyramine determination. The sensor performance was compared with previous reported results in Table 2. Compared with these studies, the established method was higher sensitivity or wider detection range by improved specificity of MOFs and enhanced adsorption property of the molecularly imprinted polymers.

Table 1. Cycling performance of the sensor tested in the samples $(n=3)$.

\begin{tabular}{cccc}
\hline Dosage $\left(\boldsymbol{\mu g} \cdot \mathbf{L}^{-\mathbf{1}}\right)$ & Measured Quantity $\left(\boldsymbol{\mu g} \cdot \mathbf{L}^{-\mathbf{1}}\right)$ & Rate of Recovery $(\%)$ & RSD $(\%)$ \\
\hline 200 & 182.96 & 91.47 & 3.7 \\
300 & 280.88 & 93.62 & 2.2 \\
400 & 346.95 & 86.74 & 1.1 \\
\hline
\end{tabular}

Table 2. Comparison of different analytical approaches for tyramine determination.

\begin{tabular}{cccc}
\hline Method & Linear Range $\left(\boldsymbol{\mu g} \cdot \mathbf{L}^{-\mathbf{1}}\right)$ & LOD $\left(\boldsymbol{\mu g} \cdot \mathbf{L}^{-\mathbf{1}}\right)$ & Reference \\
\hline HPLC & $0.05-0.25$ & 0.06 & {$[53]$} \\
DPV & $(0.685-24.69) \times 10^{3}$ & 85.05 & {$[54]$} \\
Amperometry & $(1.37-68.0) \times 10^{3}$ & 274.36 & {$[55]$} \\
Colorimetry & $5-40$ & 2 & {$[56]$} \\
QCM & $80-500$ & 61.65 & This work \\
\hline
\end{tabular}




\section{Conclusions}

In this paper, a QCM sensor based on UiO-66@MIPs were constructed and applied for tyramine detection. The material was characterized and the detection conditions were optimized, and it was applied to monitor the content of tyramine in soy sauce samples. The results showed that the sensor has the best response when the mass ratio of membrane material was $4: 1$ and the adsorption time was $16 \mathrm{~min}$. The linear response of the sensor was detected in tyramine standard solution of $80-500 \mu \mathrm{g} \cdot \mathrm{L}^{-1}$, the regression equation was obtained: $y=0.6549 x-41.185\left(R^{2}=0.9893\right)$, and its LOD was $61.65 \mu \mathrm{g} \cdot \mathrm{L}^{-1}$. The UiO-66@MIPs QCM sensor had high specificity for tyramine. At last, tyramine was detected five times in succession in soy sauce samples, and the calculated recoveries were in the range of 88.82-95.53\%. In this work, the feasibility between MIT and QCM technology were proved through preliminary research, so did the practicability of MOFs-MIPs. It is believable that the combination between MOFs and molecular imprinted technology is favorable for highly sensitive sensor system fabrication to tyramine.

Author Contributions: C.-X.Y. performed the analysis of the situation and created the original draft; N.Z. sorted out the experimental data; J.-M.L. proposed the experimental concept and revised the manuscript; G.-Z.F. gave the methodology and funded this experiment; S.W. contributed reagents/materials/analysis tools and supervised the experiment. All authors have read and agreed to the published version of the manuscript.

Acknowledgments: This work was supported by the Open Project Program of State Key Laboratory of Food Nutrition and Safety, Tianjin University of Science \& Technology (No. SKLFNS-KF-201907), and the National Natural Science Foundation of China (No. 21806083).

Conflicts of Interest: The authors declare no conflict of interest.

\section{References}

1. Mazzucco, E.; Gosetti, F.; Bobba, M.; Marengo, E.; Robotti, E.; Gennaro, M.C. High-performance liquid chromatography-ultraviolet detection method for the simultaneous determination of typical biogenic amines and precursor amino acids. Applications in food chemistry. J. Agric. Food. Chem. 2010, 58, 127-134. [CrossRef] [PubMed]

2. Antal, E.; Hendershot, P.; Batts, D.; Sheu, W.; Hopkins, N.; Donaldson, K. Linezolid, a novel oxazolidinone antibiotic: Assessment of monoamine oxidase inhibition using pressor response to oral tyramine. J. Clin. Pharmacol. 2001, 41, 552-562. [CrossRef]

3. Önal, A. A review: Current analytical methods for the determination of biogenic amines in foods. Food Chem. 2007, 103, 1475-1486. [CrossRef]

4. Özkan, A.; Atar, N.; Yola, M.L. Enhanced surface plasmon resonance (SPR) signals based on immobilization of core-shell nanoparticles incorporated boron nitride nanosheets: Development of molecularly imprinted SPR nanosensor for anticancer drug, etoposide. Biosens. Bioelectron. 2019, 130, 293-298. [CrossRef]

5. Li, Y.; Hsieh, C.-H.; Lai, C.-W.; Chang, Y.-F.; Chan, H.-Y.; Tsai, C.-F.; Ho, J.-A.A.; Wu, L.-C. Tyramine detection using PEDOT:PSS/AuNPs/1-methyl-4-mercaptopyridine modified screen-printed carbon electrode with molecularly imprinted polymer solid phase extraction. Biosens. Bioelectron. 2017, 87, 142-149. [CrossRef]

6. Bieck, P.; Antonin, K.-H. Tyramine potentiation during treatment with MAO inhibitors: Brofaromine and moclobemide vs irreversible inhibitors. J. Neural Transm. 1989, 28, 21-31.

7. Huang, K.J.; Wei, C.Y.; Liu, W.L.; Xie, W.Z.; Zhang, J.F.; Wang, W. Ultrasound-assisted dispersive liquid-liquid microextraction combined with high-performance liquid chromatography-fluorescence detection for sensitive determination of biogenic amines in rice wine samples. J. Chromatogr. A 2009, 1216, 6636-6641. [CrossRef]

8. Lapainis, T.; Scanlan, C.; Rubakhin, S.S.; Sweedler, J.V. A multichannel native fluorescence detection system for capillary electrophoretic analysis of neurotransmitters in single neurons. Anal. Bioanal. Chem. 2007, 387, 97-105. [CrossRef] [PubMed]

9. Kim, T.-K.; Lee, J.-I.; Kim, J.-H.; Mah, J.-H.; Hwang, H.-J.; Kim, Y.-W. Comparison of ELISA and HPLC methods for the determination of biogenic amines in commercial Doenjang and Gochujang. Food Sci. Biotechnol. 2011, 20, 1747-1750. [CrossRef]

10. Apetrei, I.M.; Apetrei, C. Amperometric biosensor based on polypyrrole and tyrosinase for the detection of tyramine in food samples. Sens. Actuators B Chem. 2013, 178, 40-46. [CrossRef] 
11. Luo, W.; Zhu, L.; Yu, C.; Tang, H.; Yu, H.; Li, X.; Zhang, X. Synthesis of surface molecularly imprinted silica micro-particles in aqueous solution and the usage for selective off-line solid-phase extraction of 2,4-dinitrophenol from water matrixes. Anal. Chim. Acta. 2008, 618, 147-156. [CrossRef] [PubMed]

12. Piletska, E.V.; Guerreiro, A.R.; Romero-Guerra, M.; Chianella, I.; Turner, A.P.F.; Piletsky, S.A. Design of molecular imprinted polymers compatible with aqueous environment. Anal. Chim. Acta 2008, 607, 54-60. [CrossRef] [PubMed]

13. Miao, S.S.; Wu, M.S.; Zuo, H.G.; Jiang, C.; Jin, S.F.; Lu, Y.C.; Yang, H. Core-shell magnetic molecularly imprinted polymers as sorbent for sulfonylurea herbicide residues. J. Agric. Food Chem. 2015, 63, 3634-3645. [CrossRef] [PubMed]

14. Baggiani, C.; Anfossi, L.; Giovannoli, C. Solid phase extraction of food contaminants using molecular imprinted polymers. Anal. Chim. Acta 2007, 591, 29-39. [CrossRef] [PubMed]

15. Allender, C.J.; Richardson, C.; Woodhouse, B.; Heard, C.M.; Brain, K.R. Pharmaceutical applications for molecularly imprinted polymers. Int. J. Pharm. 2000, 195, 39-43. [CrossRef]

16. Medetalibeyoğlu, H.; Manap, S.; Yokuş, Ö.A.; Beytur, M.; Kardaş, F.; Akyıldırım, O.; Özkan, V.; Yüksek, H.; Yola, M.L.; Atar, N. Fabrication of Pt/Pd nanoparticles/polyoxometalate/ionic liquid nanohybrid for electrocatalytic oxidation of methanol. J. Electrochem. Soc. 2018, 165, F338-F341. [CrossRef]

17. Kıran, T.R.; Atar, N.; Yola, M.L. A methyl parathion recognition method based on carbon nitride incorporated hexagonal boron nitride nanosheets composite including molecularly imprinted polymer. J. Electrochem. Soc. 2019, 166, H495-H501. [CrossRef]

18. Yola, M.L.; Eren, T.; Atar, N. A molecular imprinted voltammetric sensor based on carbon nitride nanotubes: Application to determination of melamine. J. Electrochem. Soc. 2016, 163, B588-B593. [CrossRef]

19. Atar, N.; Yola, M.L. Core-shell nanoparticles/two-dimensional (2D) hexagonal boron nitride nanosheets with molecularly imprinted polymer for electrochemical sensing of cypermethrin. J. Electrochem. Soc. 2018, 165, H255-H262. [CrossRef]

20. Kabir, E.R.; Rahman, M.S.; Rahman, I. A review on endocrine disruptors and their possible impacts on human health. Environ. Toxicol. Pharmacol. 2015, 40, 241-258. [CrossRef]

21. Yola, M.L.; Atar, N. Gold nanoparticles/two-dimensional (2D) hexagonal boron nitride nanosheets including diethylstilbestrol imprinted polymer: Electrochemical detection in urine samples and validation. J. Electrochem. Soc. 2018, 165, H897-H902. [CrossRef]

22. Yola, M.L.; Göde, C.; Atar, N. Molecular imprinting polymer with polyoxometalate/carbon nitride nanotubes for electrochemical recognition of bilirubin. Electrochim. Acta 2017, 246, 135-140. [CrossRef]

23. Yola, M.L.; Atar, N. Development of cardiac troponin-I biosensor based on boron nitride quantum dots including molecularly imprinted polymer. Biosens. Bioelectron. 2019, 126, 418-424. [CrossRef] [PubMed]

24. Yola, M.L.; Atar, N. Development of molecular imprinted sensor including graphitic carbon nitride/N-doped carbon dots composite for novel recognition of epinephrine. Compos. Part B Eng. 2019, 175, 107113. [CrossRef]

25. Bragazzi, N.L.; Amicizia, D.; Panatto, D.; Tramalloni, D.; Valle, I.; Gasparini, R. Quartz-crystal microbalance (QCM) for public health: An overview of its applications. Adv. Protein. Chem. Struct. Biol. 2015, 101, 149-211. [CrossRef] [PubMed]

26. Yuan, Y.J.; Han, K. Development of a real-time QCM bond-rupture system for POCT applications. IEEE Sens. J. 2016, 16, 8731-8735. [CrossRef]

27. Li, W.; Wen, X.Y.; Li, S.M.; Wang, X.; Wang, J.Z.; Tang, H. Determination of DMMP using a polymer coated QCM sensor. Adv. Mater. Res. 2012, 542-543, 959-962. [CrossRef]

28. Sankaran, S.; Panigrahi, S.; Mallik, S. Olfactory receptor based piezoelectric biosensors for detection of alcohols related to food safety applications. Sens. Actuators B Chem. 2011, 155, 8-18. [CrossRef]

29. Bakhshpour, M.; Özgür, E.; Bereli, N.; Denizli, A. Microcontact imprinted quartz crystal microbalance nanosensor for protein C recognition. Colloids Surf. B Biointerfaces 2017, 151, 264-270. [CrossRef]

30. Hussain, M.; Kotova, K.; Lieberzeit, P.A. Molecularly imprinted polymer nanoparticles for formaldehyde sensing with QCM. Sensors 2016, 16, 1011. [CrossRef]

31. Lin, T.-Y.; Hu, C.-H.; Chou, T.-C. Determination of albumin concentration by MIP-QCM sensor. Biosens. Bioelectron. 2004, 20, 75-81. [CrossRef]

32. Kayal, S.; Sun, B.; Chakraborty, A. Study of metal-organic framework MIL-101(Cr) for natural gas (methane) storage and compare with other MOFs (metal-organic frameworks). Energy 2015, 91, 772-781. [CrossRef] 
33. Wang, Y.; Zhao, D. Beyond equilibrium: Metal-organic frameworks for molecular sieving and kinetic gas separation. Cryst. Growth Des. 2017, 17, 2291-2308. [CrossRef]

34. Yang, X.; Lv, J.; Yang, Z.; Yuan, R.; Chai, Y. A sensitive electrochemical aptasensor for thrombin detection based on electroactive co-based metal-organic frameworks with target-triggering NESA strategy. Anal. Chem. 2017, 89, 11636-11640. [CrossRef]

35. Zheng, L.; Li, X.; Du, W.; Shi, D.; Ning, W.; Lu, X.; Hou, Z. Metal-organic framework derived Cu/ZnO catalysts for continuous hydrogenolysis of glycerol. Appl. Catal. B Environ. 2017, 203, 146-153. [CrossRef]

36. Nadizadeh, Z.; Naimi-Jamal, M.R.; Panahi, L. Mechanochemical solvent-free in situ synthesis of drug-loaded $\left\{\mathrm{Cu}_{2}(1,4-\mathrm{bdc})_{2} \text { (dabco) }\right\}_{\mathrm{n}} \mathrm{MOF}$ for controlled drug delivery. J. Solid State Chem. 2018, 259, 35-42. [CrossRef]

37. Piscopo, C.G.; Polyzoidis, A.; Schwarzer, M.; Loebbecke, S. Stability of UiO-66 under acidic treatment: Opportunities and limitations for post-synthetic modifications. Microporous Mesoporous Mater. 2015, 208, 30-35. [CrossRef]

38. Chavan, S.M.; Vitillo, J.; Uddin, M.J.; Bonino, F.; Lamberti, C.; Groppo, E.; Lillerud, K.; Bordiga, S. Functionalization of UiO-66 metal-organic framework and highly cross-linked polystyrene with $\mathrm{Cr}(\mathrm{CO})_{3}$ : In situ formation, stability, and photoreactivity. Chem. Mater. 2010, 22, 4602-4611. [CrossRef]

39. Schaate, A.; Roy, P.; Lippke, J.; Waltz, F.; Wiebcke, M.; Behrens, P. Modulated synthesis of Zr-based metal-organic frameworks: From nano to single crystals. Chem. Eur. J. 2011, 17, 6643-6651. [CrossRef]

40. Lu, G.; Cui, C.; Zhang, W.; Liu, Y.; Huo, F. Synthesis and self-assembly of monodispersed metal-organic framework microcrystals. Chem. Asian J. 2013, 8, 69-72. [CrossRef]

41. Arrua, R.; Peristyy, A.; Nesterenko, P.; Das, A.; D’Alessandro, D.; Hilder, E. UiO-66@SiO 2 core-shell microparticles as stationary phases for the separation of small organic molecules. Analyst 2017, 142, 517-524. [CrossRef]

42. Abid, H.; Ang, H.; Wang, S. Effects of ammonium hydroxide on the structure and gas adsorption of nanosized Zr-MOFs (UiO-66). Nanoscale 2012, 4, 3089-3094. [CrossRef]

43. Shokouhfar, N.; Aboutorabi, L.; Morsali, A. Improving the capability of UiO-66 for Cr(VI) adsorption from aqueous solutions by introducing the isonicotinate N-Oxide as the functional group. Dalton Trans. 2018, 47, 14549-14555. [CrossRef]

44. Qiu, J.; Feng, Y.; Zhang, X.; Jia, M.; Yao, J. Acid-promoted synthesis of UiO-66 for highly selective adsorption of anionic dyes: Adsorption performance and mechanisms. J. Colloid Interface Sci. 2017, 499, 151-158. [CrossRef]

45. Tang, P.; Bao, T.; Chen, Z. Novel Zn-based MOFs stationary phase with large pores for capillary electrochromatography. Electrophoresis 2016, 37, 2181-2189. [CrossRef]

46. Jiao, Y.; Liu, Y.; Zhu, G.; Hungerford, J.; Bhattacharyya, S.; Lively, R.; Sholl, D.; Walton, K. Heat-treatment of defective UiO-66 from modulated synthesis: Adsorption and stability studies. J. Phys. Chem. C 2017, 121, 23471-23479. [CrossRef]

47. Tan, Y.; Zhang, W.; Gao, Y.; Wu, J.; Tang, B. Facile synthesis and supercapacitive properties of Zr-metal organic frameworks (UiO-66). RSC Adv. 2015, 5, 17601-17605. [CrossRef]

48. Chang, S.-F.; Ayres, J.W.; Sandine, W.E. Analysis of cheese for histamine, tyramine, tryptamine, histidine, tyrosine, and tryptophane1. J. Dairy Sci. 1985, 68, 2840-2846. [CrossRef]

49. Jia, L.; Rong-Fa, G.; Xi-Ming, W.; Jian-Chu, C.; Ya-Qin, H.; Dong-Hong, L.; Xing-Qian, Y. Detection of ten biogenic amines in Chinese commercial soybean paste by HPLC. Int. J. Food Prop. 2018, 21, 1344-1350. [CrossRef]

50. Antolini, F.; Franciosini, S.; Floridi, A.; Floridi, A. An ion pair HPLC method for the determination of histamine, tyramine, tryptamine, $\beta$-phenylethylamine and their amino acid precursors in cheeses for industrial purposes. Ital. J. Food Sci. 1999, 11, 335-346.

51. Veciana-Nogués, M.T.; Mariné-Font, A.; Vidal-Carou, M.C. Changes in biogenic amines during the storage of Mediterranean anchovies immersed in oil. J. Agric. Food Chem. 1997, 45, 1385-1389. [CrossRef]

52. Şenel, E.; Yildiz, F.; Yetişemiyen, A.; Durlu-Özkaya, F.; Oztekin, S.; Şanli, E. Evaluation of the biogenic amine content and some chemical and microbiological properties of urfa and van herby cheeses. Kafkas Üniversitesi Veteriner Fakültesi Dergisi 2012, 18, 537-544. [CrossRef]

53. Calbiani, F.; Careri, M.; Elviri, L.; Mangia, A.; Pistarà, L.; Zagnoni, I. Rapid assay for analyzing biogenic amines in cheese: Matrix solid-phase dispersion followed by liquid chromatography-electrospray-tandem mass spectrometry. J. Agric. Food Chem. 2005, 53, 3779-3783. [CrossRef] 
54. Apetrei, I.; Apetrei, C. The biocomposite screen-printed biosensor based on immobilization of tyrosinase onto the carboxyl functionalised carbon nanotube for assaying tyramine in fish products. J. Food Eng. 2015, 149, 1-8. [CrossRef]

55. Bóka, B.; Adányi, N.; Virág, D.; Sebela, M.; Attila, K. Spoilage detection with biogenic amine biosensors, comparison of different enzyme electrodes. Electroanalysis 2012, 24, 181-186. [CrossRef]

56. Teepoo, S.; Promta, A.; Phapugrangkul, P. A Competitive colorimetric immunosensor for detection of tyramine in fish samples. Food Anal. Methods 2019, 12, 1886-1894. [CrossRef]

(C) 2020 by the authors. Licensee MDPI, Basel, Switzerland. This article is an open access article distributed under the terms and conditions of the Creative Commons Attribution (CC BY) license (http://creativecommons.org/licenses/by/4.0/). 